patients gynecologic examination revealed pigmented lesion from which a biopsy was taken. Histologic examination indicated the diagnose of a melanoma. The mean tumor's size was $3.5 \pm 2.1 \mathrm{~cm}$ with clinically negative nodes and a mean Breslow depth $11.2 \pm 8.7 \mathrm{~mm}$. PET-CT was solicited as presurgical imaging workup.

According to the AJCC Cancer Staging Manual, 3/7 $(42.8 \%)$ of patients were diagnosed at IIC stage, $1 / 7(14.3 \%)$ at IIIB stage, $2 / 7(28.6 \%)$ at IIIC stage and $1 / 7(14.3 \%)$ at IV stage. The treatment was surgery: $3 / 7(42.8 \%)$ of patients underwent wide local excision and $4 / 7$ (57.2\%) of patients underwent hemivulvectomy. Negative margins were obtained in $4 / 7(57.2 \%)$ of surgerys. Sentinel lymph node (SLN) biopsy was performed in $5 / 7(71.4 \%)$ of patients and SLN confirmed nodal metastases in $4 / 5(80 \%)$ of patients. Immunotherapy was the adjuvant treatment for patients with $\geq$ IIIB stage (4/ 7 ), one of them combined with radiotherapy.

During the follow up, 5/7 (71.4\%) of patients relapsed. The mean time to relapsed was $5.5 \pm 2.3$ months. The treatment for the recurrences was $3 / 5(60 \%)$ cytoreductive surgery combined with immunotherapy in two patients. One woman $(20 \%)$ received a combination of radiotherapy and immunotherapy and other woman (20\%) received palliative treatment. At the time of last follow up, 4/7 (57.2\%) women died because of melanoma, $2 / 7(28.6 \%)$ was tumor free and $1 / 7$ $(14.3 \%)$ was alive with the disease.

Conclusion* As reported in literature, primary vulvar melanomas have biologically aggressive characteristics. The treatment consists of a surgery with appropriate free surgical margins. However, early recognition is what brings the maximal benefit to survival.

\section{V-Y FLAP RECONSTRUCTION FOR VULVAR CANCER RECURRENCE AFTER PRIMARY RADIOTHERAPY}

${ }^{1} S$ Fernandez-Gonzalez* ${ }^{1} \mathrm{C}$ Ortega, ${ }^{1} \mathrm{~L}$ Marti, ${ }^{2} \mathrm{JO}$ Bermejo, ${ }^{2} \mathrm{JA}$ Palacin, ${ }^{3} \mathrm{Y}$ Perez, ${ }^{3} \mathrm{~S}$ Martorell, ${ }^{3} \mathrm{M}$ Alvarez, ${ }^{1} \mathrm{M}$ Barahona, ${ }^{1} \mathrm{~J}$ Ponce. ${ }^{1}$ University Hospital of Bellvitge (IDIBELL), Gynecology, L'Hospitalet de Llobregat, Spain; ${ }^{2}$ University Hospital of Bellvitge (IDIBELL), Plastic and reconstructive surgery, L'Hospitalet de Llobregat, Spain; ${ }^{3}$ University Hospital of Bellvitge (IDIBELL), Nursery, L'Hospitalet de Llobregat, Spain

\subsection{6/ijgc-2021-ESG0.628}

Introduction/Background* Vulvar cancer is a rare disease specially in young women. When a large tumour is diagnosed, reconstruction flap should be considered in order to achieve the best cosmetic and functional result.

Methodology This surgical video shows the excision of a perianal recurrence secondary to a vulvar cancer after primary radiotherapy. Rectal amputation and terminal colostomy were performed through a laparoscopic approach. A bilateral V-Y flap was designed for perineal reconstruction. This procedure was recorded step by step.

Result(s)* A 47 years old woman was diagnosed with perianal VIN III. It measured $25 \mathrm{~mm}$ and had no extraperineal disease. Primary radiotherapy was indicated due to the invasion of anal mucosa. After 6 months of treatment, perianal recurrence of scamous vulvar carcinoma was diagnosed. Consequently, wide surgical excision, anal amputation, bilateral V-Y flap reconstruction and terminal colostomy were performed under lithotomy position and general anaesthesia. Despite surgical wound infection by pseudomonas and partial dehiscence, very good cosmetic and functional results were achieved.

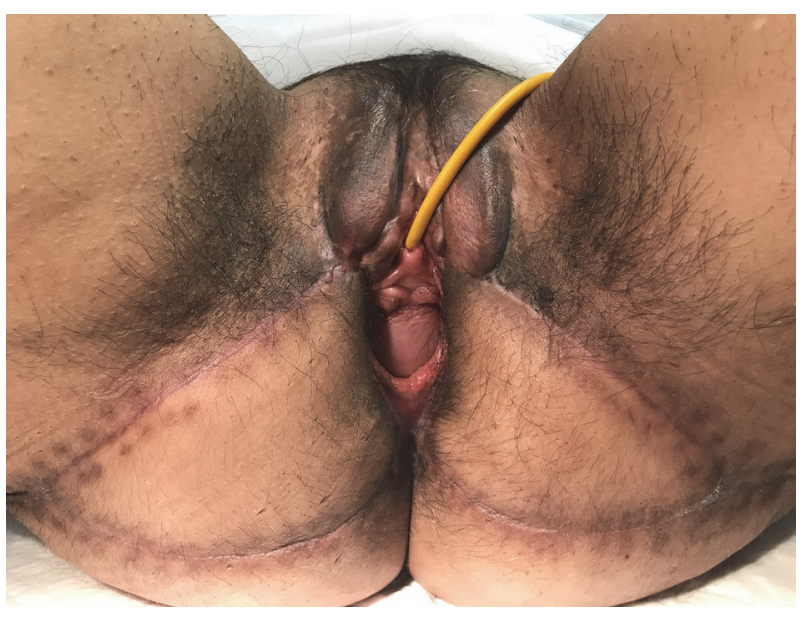

Abstract 1021 Figure 1

Conclusion* V-Y flap reconstruction may be a good option for the treatment of perineal defects that are too large for primary intention wound closure.

\section{TRANSINGUINAL PELVIC LYMPHADENECTOMY IN VULVAR CANCER PATIENTS (TRIPLE STUDY): A SINGLE- INSTITUTION, PROSPECTIVE, PILOT STUDY}

${ }^{1} \mathrm{SM}$ Fragomeni*, ${ }^{2} \mathrm{~L}$ Della Corte, ${ }^{1} \mathrm{C}$ Conte, ${ }^{1} \mathrm{~A}$ Federico, ${ }^{3} \mathrm{~B}$ Marinucci, ${ }^{4} \mathrm{~L}$ Tagliaferri, ${ }^{5} \mathrm{~S}$ Gentileschi, ${ }^{1} \mathrm{G}$ Corrado, ${ }^{6}$ I Romito, ${ }^{3} \mathrm{~A}$ Zampolini Faustini, ${ }^{1} \mathrm{LC}$ Turco, ${ }^{1} \mathrm{~V}$ Gallotta, ${ }^{1,3} \mathrm{~A}$ Fagotti, ${ }^{1,3} \mathrm{G}$ Scambia, ${ }^{3,6} \mathrm{G}$ Garganese. ${ }^{1}$ Fondazione Policlinico Universitario A. Gemelli IRCCS, Unità di Ginecologia Oncologica, Dipartimento Scienze della Salute della Donna, del Bambino e di Sanità Pubblica, Rome, Italy; ${ }^{2}$ University of Naples Federico II, Department of Neuroscience, Reproductive Sciences and Dentistry, School of Medicine, Naples, Italy; ${ }^{3}$ Università Cattolica del Sacro Cuore, Istituto di Clinica Ostetrica e Ginecologica, Rome, Italy; ${ }^{4}$ Fondazione Policlinico Universitario A. Gemelli IRCCS, Unità Operativa Complessa di Radioterapia, Dipartimento di Scienze Radiologiche, Radioterapiche ed Ematologiche, Rome, Italy; ${ }^{5}$ Fondazione Policlinico Universitario A. Gemelli IRCCS, Unità di Chirurgia Plastica, Dipartimento Scienze della Salute della Donna, del Bambino e di Sanità Pubblica, Rome, Italy; ${ }^{6}$ Mater Olbia Hospital, Gynecology and Breast Care Center, Olbia, Italy

\subsection{6/ijgc-2021-ESG0.629}

Introduction/Background* Vulvar cancer (VC) is a lymphotropic disease. The preferred lymphatic pathway involves the inguinofemoral lymph nodes (IFLNs), followed by the pelvic lymph nodes (PLNs). In selected cases, surgical approach to PLNs may be the first choice when histologic confirmation is required for suspicious involvement on imaging or when radiation therapy is out of indication (e.g., in cases of previous radiation therapy or in specific histotypes).

Given the anatomic continuity between the inguinal and pelvic sites, a novel retrograde trans-inguinal pelvic access was explored to provide concomitant lymphadenectomy, with a single skin incision and no change in surgical position or instruments.

Our objective was to evaluate the feasibility and safety of the extraperitoneal trans-inguinal novel approach to pelvic lymphadenectomy (TRIPLE).

Methodology All consecutive patients referred to our Institution in the last 18 months, affected by primary/recurrent VC, candidate to concomitant groin and pelvic lymph node surgery were included. After conventional IFLN dissection, ipsilateral extraperitoneal trans-inguinal pelvic lymphadenectomy (TRIPLE) was performed. Clinical data, type of treatment, 